\title{
Short- and long-term outcomes of incarcerated inguinal hernias repaired by Lichtenstein technique
}

\author{
Andrzej Wysocki, Marcin Strzałka, Marcin Migaczewski, Piotr Budzyński \\ $2^{\text {nd }}$ Department of General Surgery, Medical College Jagiellonian University, Krakow, Poland
}

Videosurgery Miniinv 2014; 9 (2): 196-200

DOI: $10.5114 /$ wiitm.2014.41630

\begin{abstract}
Introduction: The use of tension-free inguinal hernia repair techniques using commercially available implants is now rather common. However, it is widely accepted that the use of biomaterials should be limited to non-infected surgical fields. As such, most current studies pertain to the application of various implants during the surgical repair of uncomplicated hernias.

Aim: To compare the short-and long-term outcomes of incarcerated inguinal hernia repair using the Lichtenstein or Bassini technique.

Material and methods: Between 1997 and 2012, 107 patients were operated on an emergency basis due to the incarceration of inguinal hernias - 105 subjects were included for further analysis in our study.

Results: Postoperative complications were observed in 13 out of the 84 (15.5\%) patients subjected to Lichtenstein repair. In 9 of these patients (10.7\%), morbidity was associated with the surgical wound. In 2 cases (2.4\%), a small inflammatory infiltration was observed and resolved within a few days. Serous fluid accumulation within the wound was observed in 3 patients (3.6\%), but the fluid was successfully drained by puncture. Finally, hematoma formed in 4 cases (4.8\%). In total, 4 complications (19\%) were recorded in the group of 21 patients who were operated on with the Bassini technique. In 3 of these cases (14.3\%), the complications were related to suppuration of the surgical wound.

Conclusions: Polypropylene mesh may be safely implanted during the repair of incarcerated hernia and this approach is reflected by satisfactory long-term outcomes.
\end{abstract}

Key words: incarcerated inguinal hernias, Lichtenstein technique.

\section{Introduction}

The use of tension-free inguinal hernia repair techniques using commercially available implants is now rather common [1]. However, it is widely accepted that the use of biomaterials should be limited to non-infected surgical fields. As such, most current studies pertain to the application of various implants during the surgical repair of uncomplicated hernias. Although hernia incarceration is one of the more frequent causes of bowel strangulation, little attention is paid to the use of biomaterials for this indication, and studies on the postoperative and long-term outcomes in these patients are sparse.

\section{Aim}

The aim of this study was to compare the shortand long-term outcomes of incarcerated inguinal hernia repair using the Lichtenstein or Bassini technique. Moreover, we analyzed how the attitudes to the ad hoc application of biomaterials in complicated cases of inguinal hernia have changed over time. 


\section{Material and methods}

Between 1997 and 2012, 107 patients were operated on an emergency due to the incarceration of inguinal hernias. Two patients died during the initial $24 \mathrm{~h}$ after surgery (due to massive myocardial infarct and shock). Therefore, 105 subjects were included for further analysis in our study. The age of the patients ranged from 21 to 95 years, with a mean age of $63.35 \pm 18.15$. Most patients $(n=84)$ were operated on by means of Lichtenstein repair using a monofilament or double filament polypropylene patch. Implants were not used in the remaining 21 patients. In all of the cases, the operator chose which method of hernia repair to use based on his or her experience and individual preferences.

Amongst the patients who were subjected to Lichtenstein repair, 69 cases of incarceration were related to primary hernia, and 15 cases to recurrent hernia. The hernial sac contained the small intestine in 49 patients, the large intestine in 16 patients, both the small and large intestines in 3 patients, and the omentum in 16 patients (Table I). Two patients underwent intestinal resection, whereas omental resection was required in 7 cases (Table II). In 1 case, a necrotic epiploic appendix was resected, while the resection of an incarcerated Meckel's diverticulum was required in another case (Table II). Three patients died due to reasons unrelated to hernia incarceration on the $7^{\text {th }}, 15^{\text {th }}$ and $50^{\text {th }}$ days of hospitalization.

All patients were given intravenous antibiotic therapy. The treatment was started intra-operatively and, depending on the local status, continued for 2 to 5 days (in case of inflammatory changes within the wound). The class of antibiotics used changed over time following the recommendations of the hospital committee for nosocomial infection control. The treatment protocols included aminoglycosides

Table I. Content of hernial sac

\begin{tabular}{|lcc|}
\hline Variable & Without mesh & With mesh \\
\hline Small intestine & 12 & 49 \\
\hline Large intestine & 3 & 16 \\
\hline $\begin{array}{l}\text { Large and small } \\
\text { intestine }\end{array}$ & 0 & 4 \\
\hline Omentum (only) & 7 & 16 \\
\hline All & 22 & 85 \\
\hline
\end{tabular}

and first and second generation cephalosporins combined with metronidazole.

Amongst the 21 patients who were subjected to the tension techniques (18 primary hernias and 3 recurrent hernias), incarceration included the small intestine in 11 patients, the large intestine in 3 patients, and the omentum in 7 patients (Table I). Intestinal resection was required in 6 cases, while omental resection was required in 2 cases (Table II).

The proportions of cases operated on by either method were calculated separately for each of the following three time periods: 1997-2000, 2001-2004, and 2005-2012.

Further ambulatory follow-up was available for $88.8 \%$ of the 102 patients. The duration of follow-up ranged from 1 to 12 years (mean: $61.4 \pm 38.9$ months).

\section{Results}

Postoperative complications were observed in 13 out of the 84 (15.5\%) patients subjected to Lichtenstein repair. In 9 of these patients (10.7\%), morbidity was associated with the surgical wound. In 2 cases (2.4\%), a small inflammatory infiltration was observed and resolved within a few days. Serous fluid accumulation within the wound was observed in 3 patients (3.6\%), but the fluid was successfully drained by puncture. Finally, hematoma formed in 4 cases $(4.8 \%)$.

No postoperative morbidities related to synthetic material implantation were noted in 76 out of the 84 (90.5\%) patients.

Other morbidities included exacerbation of coronary heart disease $(n=1)$, postoperative pneumonia

Table II. Type of surgical procedure

\begin{tabular}{|lcc|}
\hline Variable & Without mesh & With mesh \\
\hline $\begin{array}{l}\text { Release of the bowel } \\
\text { loop }\end{array}$ & 8 & 65 \\
\hline Release of the omentum & 5 & 9 \\
\hline $\begin{array}{l}\text { Intestinal resection } \\
\text { Resection of epiploic } \\
\text { appendix }\end{array}$ & 7 & 2 \\
\hline $\begin{array}{l}\text { Resection of Meckel's } \\
\text { diverticulum }\end{array}$ & 0 & 1 \\
\hline Omental resection & 2 & 7 \\
\hline All & 22 & 85 \\
\hline
\end{tabular}


$(n=1)$, growing ascites associated with decompensated liver cirrhosis $(n=1)$, and urinary retention $(n=1)$.

In total, 4 complications (19\%) were recorded in the group of 21 patients who were operated on with the Bassini technique. In 3 of these cases (14.3\%), the complications were related to suppuration of the surgical wound. Moreover, there was 1 fatal case (4.8\%) due to stroke during the postoperative period.

During further follow-up, no cases of recurrent hernia or late inflammatory complications were noted in any of the subjects belonging to both groups.

During the initial period covered by this study, the tension and tension-free techniques of hernia repair were used with a similar frequency. During the second and third analyzed period, however, the proportion of patients operated on using tension techniques decreased to $9 \%$ and $3 \%$, respectively.

\section{Discussion}

For decades, the method described by Bassini remained the gold standard in inguinal hernia repair and was used by generations of surgeons. However, the limitations of this technique became obvious with time, particularly in regards to the high recurrence rates noted during long-term observations (recurrence rates would sometimes reach over $20 \%$, sometimes even 30\%) [2, 3]. In turn, a dramatic decrease in recurrence rates was observed with the growing popularity of surgical procedures utilizing modern and more advanced synthetic materials.

In comparison to numerous published works pertaining to inguinal hernias, it seems that little attention is paid to hernia incarceration. Kingsnorth, using MEDLINE and EMBASE, analyzed 135 published papers in his systematic review of the literature (published in The Lancet). Out of these 135 papers, only 2 papers dealt with hernias complicated by incarceration (implants were used in both cases) $[4,5]$.

Polypropylene mesh is the most frequently used material in tension-free surgery. This mesh stimulates fibroblasts, leading to the formation of a strong connective tissue scar. This scar significantly improves the durability of the fasciomuscular layer formed during surgery. The microscopic structure of the mesh patch plays an important role, particularly in regards to the diameter of the mesh openings and filaments. Macrophages can freely penetrate into mono- or double filament meshes with openings larger than $75 \mathrm{mi}-$ crons, eliminating potential infections. This finding has important practical implications since implants with this type of structure enable the control of potential infections, particularly important in surgery performed within an infected field.

Pans et al. [6] were the first to describe the use of implants during the repair of incarcerated hernias. In their paper from 1997, these authors confirmed that the implantation of a synthetic patch is safe and not associated with the risk of infection [6]. A limited number of papers dealing with this subject have been published since then. Initially, the question of using implants within an infected surgical field raised serious controversies. With time, however, there has been a gradual increase in the number of papers suggesting that there is a low risk for local infection and that there are other advantages related to the use of this technique [7]. Closing the openings of complicated umbilical hernias with mesh in liver cirrhosis patients was reflected by similar postoperative morbidity rates as in patients operated on without mesh, but recurrence rates were found to be markedly lower [8]. Also, it was found that bowel resection was not a factor in increasing the rate of postoperative complications and recurrences in cases of prosthetic repair of acutely incarcerated inguinal hernias [9]. There is increasing evidence of favorable results after mesh patch implantation in cases with simultaneous large bowel surgery or peristomal hernia repair [10-15]. In spite of repeated concerns, failure rates due to mesh infection are very low. There also are a few case reports pertaining to the laparoscopic repair of incarcerated internal and external hernias whose openings were closed using mesh. The successful outcome of these cases further supports the hypothesis that the risk for potential infection of implanted biomaterial is low [16-20]. Growing evidence not only supports the application of such prostheses $[21,22]$, but also raises doubts about previous recommendations which limited the use of synthetic patches in infected surgical fields.

None of the fatal cases from among our patients were directly associated with the surgical technique applied. The use of the tension-free technique does not seem to influence mortality in patients who are operated on due to hernia incarceration; however, this technique has a marked influence on long-term surgical outcomes since it significantly decreases recurrence rates [23].

There are ongoing discussions pertaining to the prophylactic use of antibiotics during and after sche- 
duled tension-free hernial repairs [24]. A systematic review of the literature suggests that such an approach is reasonable [25-27]. However, this attitude is not widely accepted [28]. In case of incarceration, a local infection may be expected whenever the ischemic intestine or omentum is entrapped inside the hernial sac. This leads to massive invasion of the surgical field by highly virulent gastrointestinal microflora. In our opinion, the use of antibiotics in such circumstances is a vital component of patient management. Therefore, all of our patients received ultra-potent antibiotic therapy intraoperatively and for 2 to 5 days thereafter (the class of antibiotics used changed over time along with compulsory intra-hospital standards of nosocomial infection control).

Existing controversies associated with the use of synthetic prostheses during incarcerated hernia repair have resulted in the search for alternative materials. Small intestinal submucosa (SIS) is one such material, commercially available as Surgisis ${ }^{\circledR}$ [29]. In an interesting study, SIS was implanted laparoscopically in 116 patients who underwent hernial repair performed in an infected or potentially infected environment. The preliminary results achieved using this costly technique are highly promising. However, due to the character of SIS, further clinical research (including long-term observation) is needed before final conclusions may be formulated on the potential usefulness of this material.

Our long-term experience [30] suggests that the ad hoc closure of an incarcerated hernia opening with monofilament or double filament polypropylene mesh is safe and associated with low morbidity. Local complications in the form of wound suppuration or peripheral necrosis were observed in less than $2.5 \%$ of subjects. These complications, although successfully controlled by expectant treatment during hospitalization, may significantly affect long-term outcomes. However, no negative late sequelae of these postoperative complications were observed in our patients.

This study was not a scheduled clinical trial but rather a clinical observational study. However, this limitation is compensated by our growing experience due to the increasing number of cases subjected to Lichtenstein repair. The infrequency of inflammatory complications, along with the satisfactory postoperative and long-term outcomes, has gradually convinced our operators to use this technique during the repair of complicated inguinal hernias.
This attitude has been confirmed by the gradual decrease in the frequency of surgery performed using the Bassini technique. As a result, during the final 6-year period analyzed, only one case was operated on using this technique.

The number of patients subjected to the Bassini procedure with at least one year of follow-up is simply too small to allow us to draw conclusions regarding the long-term outcomes of this technique, despite the lack of recurrences. The group of patients who underwent the Lichtenstein repair (and who were available for follow-up) was markedly larger. Neither recurrent hernias nor late inflammatory complications were observed during long-term follow-up of this cohort. In view of the large size of this group, we may conclude that the repair of complicated inguinal hernia with the Lichtenstein technique is safe and associated with satisfactory long-term outcomes, despite potential contaminations of the surgical field [31, 32].

\section{Conclusions}

Polypropylene mesh may be safely implanted during the repair of incarcerated hernia and this approach is reflected by satisfactory long-term outcomes.

\section{References}

1. Pesta W, Kurpiewski W, Łuba M, et al. Single incision laparoscopic surgery transabdominal pre-peritoneal hernia repair case report. Videosurgery Miniinv 2012; 7: 137-9.

2. Beets GL, Oosterhuis KJ, Go PM, et al. Long term follow up (12-15 years) of a randomized controlled trial comparing Bassini-Stetten, Shouldice, and high ligation with narrowing of the internal ring for primary inguinal hernia repair. J Am Coll Surg 1997; 185: 352-7.

3. Mückter H, Reuters G, Vogel W. Bassini and Shouldice repair of inguinal hernia. A retrospective comparative study. Chirurg 1994; 65: 121-6.

4. Kingsnorth A, LeBlanc K. Hernias: inguinal and incisional. Lancet 2003; 362: 1561-71.

5. Zhao G, Gao P, Ma B, et al. Open mesh techniques for inguinal hernia repair: a meta-analysis of randomized controlled trials. Ann Surg 2009; 250: 35-42.

6. Pans A, Desaive C, Jacquet N. Use of a preperitoneal prosthesis for strangulated groin hernia. Br J Surg 1997; 84: 310-2.

7. Papaziogas B, Lazaridis C, Makris J, et al. Tension-free repair versus modified Bassini technique (Andrews technique) for strangulated inguinal hernia: a comparative study. Hernia 2005; 9: 156-9.

8. Ammar SA. Management of complicated umbilical hernias in cirrhotic patients using permanent mesh: randomized clinical trial. Hernia 2010; 14: 35-8. 
9. Atlia K, Guler S, Inal A, et al. Prothetic repair of acutely incarcerated groin hernias: a prospective clinical observational cohort study. Langensbeck Arch Surg 2010; 395: 563-8.

10. Birolini C, Utiyama EM, Rodrigues AJ Jr, Birolini D. Elective colonic operation and prosthetic repair of incisional hernia: does contamination contraindicate abdominal wall prosthesis use? J Am Coll Surg 2000; 191: 366-72.

11. Israelsson LA. Parastomal hernias. Surg Clin North Am 2008; 88: $113-25$.

12. Janes A, Cengiz Y, Israelsson LA. Randomized clinical trial of the use of a prosthetic mesh to prevent parastomal hernia. $\mathrm{Br}$ J Surg 2004; 91: 280-2.

13. Kelly ME, Behrman SW. The safety and efficacy of prosthetic hernia repair in clean-contaminated and contaminated wounds. Am Surg 2002; 68: 524-8.

14. Steele SR, Lee P, Martin MJ, et al. Is parastomal hernia repair with polypropylene mesh safe? Am J Surg 2003; 185: 436-40.

15. Vix J, Meyer C, Rohr S, Bourtoul C. The treatment of incisional and abdominal hernia with a prosthesis in potentially infected tissues - a series of 47 cases. Hernia 1997; 1: 157.

16. Ferzli GS, Shapiro K, DeTurris SV, et al. Laparoscopic extraperitoneal approach to acutely incarcerated inguinal hernia. Surg Endosc 2004; 18: 228-31.

17. Legnani GL, Rasini M, Pastori S, Sarli D. Laparoscopic trans-peritoneal hernioplasty (TAPP) for the acute management of strangulated inguino-crural hernias: a report of nine cases. Hernia 2008; 12: 185-8.

18. Bessa SS, Katri KM, Abdel-Salam WN, Abdel-Baki NA. Early results from the use of the Lichtenstein repair in the management of strangulated groin hernia. Hernia 2007; 11: 239-42.

19. Elsebae MM, Nasr M, Said M. Tension-free repair versus Bassini technique for strangulated inguinal hernia: a controlled randomized study. Int J Surg 2008; 6: 302-5.

20. Rebuffat C, Galli A, Scalambra MS, Balsamo F. Laparoscopic repair of strangulated hernias. Surg Endosc 2006; 20: 131-4.

21. Karatepe O, Adas G, Battal M, et al. The comparison of preperitoneal and Lichtenstein repair for incarcerated groin hernias: a randomised controlled trial. Int J Surg 2008; 6: 189-92.

22. Lohsiriwat V, Sridermma W, Akaraviputh T, et al. Surgical outcomes of Lichtenstein tension-free hernioplasty for acutely incarcerated inguinal hernia. Surg Today 2007; 37: 212-4.

23. Derici H, Unalp H R, Nazli O, et al. Prosthetic repair of incarcerated inguinal hernias: is it reliable method? Langensbeck Arch Surg 2010; 395: 575-9.

24. Dąbrowiecki S, Pierściński S, Szczęsny W. The Glubran 2 glue for mesh fixation in Lichtenstein's hernia repair: a double-blind randomized study. Videosurgery Miniinv 2012; 7: 96-104.

25. Sanabria A, Domínguez LC, Valdivieso E, Gómez G. Prophylactic antibiotics for mesh inguinal hernioplasty: a meta-analysis. Ann Surg 2007; 245: 392-6.

26. Celdrán A, Frieyro O, de la Pinta JC, et al. The role of antibiotic prophylaxis on wound infection after mesh hernia repair under local anesthesia on an ambulatory basis. Hernia 2004; 8: 20-2.

27. Ríos A, Rodríguez JM, Munitiz V, et al. Antibiotic prophylaxis in incisional hernia repair using a prosthesis. Hernia 2001; 5: 148-52.

28. Aufenacker TJ, Koelemay MJ, Gouma DJ, Simons MP. Systematic review and meta-analysis of the effectiveness of antibiotic pro- phylaxis in prevention of wound infection after mesh repair of abdominal wall hernia. Br J Surg 2006; 93: 5-10.

29. Franklin ME Jr, Treviño JM, Portillo G, et al. The use of porcine small intestinal submucosa as a prosthetic material for laparoscopic hernia repair in infected and potentially contaminated fields: long-term follow-up. Surg Endosc 2008; 22: 1941-6.

30. Wysocki A, Poźniczek M, Krzywoń J, et al. Lichtenstein repair for incarcerated groin hernias. Eur J Surg 2002; 168: 452-4.

31. Pielaciński K, Szczepanik AB, Wróblewski T. Effect of mesh type, surgeon and selected patients' characteristics on the treatment of inguinal hernia with the Lichtenstein technique. Randomized trial. Videosurgery Miniinv 2013; 8: 99-106.

32. Bierca J, Kosim A, Kołodziejczak M, et al. Effectiveness of Lichtenstein repairs in planned treatment of giant inguinal hernia - own experience. Videosurgery Miniinv 2013; 8: 36-42.

Received: 5.07.2013, accepted: 5.11.2013. 